\title{
Atrial fibrillation: to map or not to map?
}

\author{
A. Yaksh • C. Kik • P. Knops • J. W. Roos-Hesselink • \\ A. J. J. C. Bogers • F. Zijlstra • M. Allessie • \\ N. M. S. de Groot
}

Published online: 16 October 2013

(C) The Author(s) 2013. This article is published with open access at Springerlink.com

\begin{abstract}
Isolation of the pulmonary veins may be an effective treatment modality for eliminating atrial fibrillation (AF) episodes but unfortunately not for all patients. When ablative therapy fails, it is assumed that AF has progressed from a trigger-driven to a substrate-mediated arrhythmia. The effect of radiofrequency ablation on persistent AF can be attributed to various mechanisms, including elimination of the trigger, modification of the arrhythmogenic substrate, interruption of
\end{abstract}

A. Yaksh • P. Knops

Department of Cardiology, Thoraxcentrum, Ba 579 Erasmus Medical

Center, 's Gravendijkwal 230, 3015 CE Rotterdam, the Netherlands

C. Kik · A. J. J. C. Bogers

Department of Cardiothoracic Surgery, Thoraxcentrum, BD-557 Erasmus Medical Center, 's Gravendijkwal 230, 3015 CE Rotterdam, the Netherlands

J. W. Roos-Hesselink

Department of Cardiology, Thoraxcentrum, Ba 583A Erasmus Medical Center, 's Gravendijkwal 230, 3015 CE Rotterdam, the Netherlands

\section{F. Zijlstra}

Department of Cardiology, Thoraxcentrum, BA-593 Erasmus Medical Center, 's Gravendijkwal 230, 3015 CE Rotterdam, the Netherlands

M. Allessie • N. M. S. de Groot

Department of Cardiology, Thoraxcentrum, BA-597 Erasmus Medical Center, 's Gravendijkwal 230, 3015 CE Rotterdam, the Netherlands

\section{Allessie}

Department of Physiology, Cardiovascular Research

Institute Maastricht, Maastricht, the Netherlands

N. M. S. de Groot $(\bowtie)$

Unit Translational Electrophysiology, Department of Cardiology,

Erasmus Medical Center, Thorax Center, 's Gravendijkwal 230,

3015 CE Rotterdam, the Netherlands

e-mail:nmsdegroot@yahoo.com crucial pathways of conduction, atrial debulking, or atrial denervation. This review discusses the possible effects of pulmonary vein isolation on the fibrillatory process and the necessity of cardiac mapping in order to comprehend the mechanisms of AF in the individual patient and to select the optimal treatment modality.

Keywords Atrial fibrillation · Pulmonary vein isolation . Cardiac surgery $\cdot$ Cardiac mapping

Atrial fibrillation (AF) is at this moment a major subject of interest for both clinicians and scientists. The interest for $\mathrm{AF}$ is partly the result of the fact that $\mathrm{AF}$ is the most common arrhythmia and therefore frequently encountered in daily clinical practice. As it is more common in the elderly $(5 \%$ in subjects $>60$ years), the incidence of AF will only continue to rise due to ageing of the population. Subjects with AF represent a spectrum of patients with arrhythmia episodes varying in duration, number, pattern of onset, triggers and mode of termination. In addition, the arrhythmia presentation itself can also change over time. Despite the variable clinical presentation, AF is characterised by an irregular heartbeat, stasis of blood flow in the left atrial appendage which increases the vulnerability to thromboembolism, and loss of the atrial kick that may compromise cardiac haemodynamics. Hence, these severe sequelae of AF require effective treatment [1-4].

In 1998, Haïssaguere et al. demonstrated that paroxysms of $\mathrm{AF}$ can be triggered by ectopic foci mainly located within the pulmonary veins (PV) [5]. Electrical isolation of the pulmonary veins (PVI) has since then been regarded as a potentially curative therapy and is nowadays indicated as a class Ia treatment modality for patients with symptomatic paroxysmal AF in whom antiarrhythmic drug therapy has failed [6]. Examples of various ablation technologies are demonstrated in Fig. 1. Numerous studies have provided evidence that PVI 

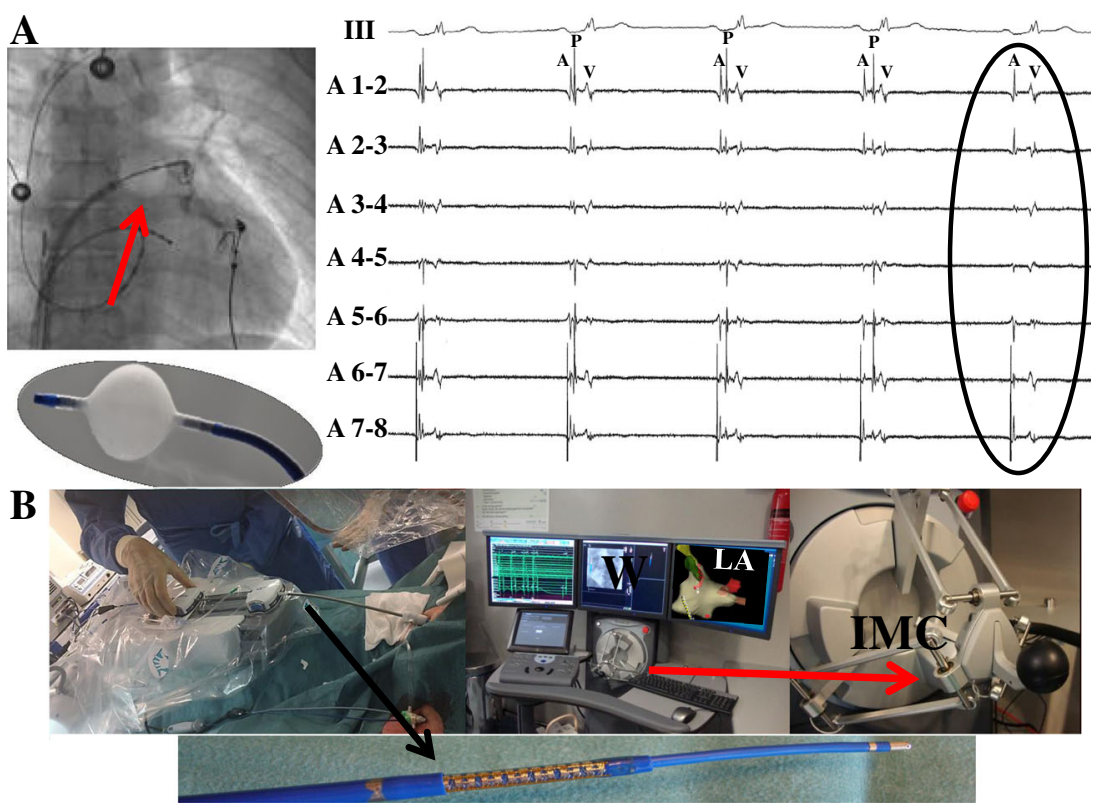

Fig. 1 Pulmonary vein isolation technologies. Isolation of the pulmonary veins using cryothermal energy (panel a) or radiofrequency energy (panel b). Panel a: A cryothermal balloon catheter (red arrow) is positioned into the left superior pulmonary vein and circumferential ostial ablation results in the disappearance of PV potentials $(P)$. Panel $\mathbf{b}$ : An endovascular, remote, steerable, radiofrequency ablation catheter is positioned in the left

can be an effective treatment modality, but unfortunately not for all patients [7-9]. Miyazaki et al. studied AF recurrences after PVI in 574 patients (paroxysmal AF: 452, persistent AF: 122). Twenty-seven months after the first PVI, 376 patients maintained sinus rhythm without the aid of antiarrhythmic drugs (paroxysmal AF: 304, persistent AF: 72). The other patients needed multiple (maximum of 4 ) redo procedures. Early recurrences of AF after 6 months revealed that they were mainly due to recovered PV conduction, whereas recurrences after 1 year were mainly caused by non-pulmonary vein triggers [7]. Kottkamp et al. reported the long-term outcome of ablative therapy in 80 patients with paroxysmal AF and 20 patients with persistent AF. Seven-day Holter recordings were taken before ablation, within $1 \mathrm{~h}$ after ablation and 3, 6 and 12 months after ablation. In patients with paroxysmal AF, AF episodes decreased over time (74\%). In patients with persistent $\mathrm{AF}$ and recurrences after ablation, the number of $\mathrm{AF}$ episodes increased over time but the duration of these $\mathrm{AF}$ episodes diminished. After a follow-up period of 12 months, only $22 \%$ of the patients with longstanding persistent AF (LsPAF) were free of AF episodes. From these findings the authors suggested that PV trigger elimination did not play a role in abolishing LsPAF and that ablation might only have modified the arrhythmogenic substrate [10].

Several studies were conducted to examine whether PVI was actually more effective than antiarrhythmic drug therapy in eliminating AF episodes. The Medical ANtiarrhythmic superior pulmonary vein. The radiofrequency ablation catheter in a steerable sheath is attached to a robotic arm (red arrow), which is controlled from a workstation $(W)$. The workstation is equipped with an instinctive motion controller $(I M C)$ which provides tactile feedback. Navigation with the robotic arm is facilitated by a three-dimensional electroanatomical map of the left atrium $(L A)$

Treatment or Radiofrequency Ablation in Paroxysmal Atrial Fibrillation (MANTRA-AF) trial was the first large randomised multicentre trial, comparing the efficacy of PVI versus antiarrhythmic drug therapy. Altogether, 294 patients were enrolled over a period of 3 years; 148 patients were treated with antiarrhythmic drug therapy and 146 underwent pulmonary vein isolation. In order to assess the AF burden, continuous rhythm monitoring was performed for 7 days at baseline, and 3, 6, 12, 18 and 24 months after initiation of therapy. A significant difference in AF burden was found between the PVI and AAD group at 24-month follow-up ( $9 \%$ vs. $18 \%$, respectively; $p=0.007$ ). However, the cumulative $\mathrm{AF}$ burden during the entire follow-up period revealed no significant difference between the two groups (13\% and $19 \%$, respectively; $p=0.10$ ) [11].

\section{Effects of the pulmonary vein isolation procedure}

Why and when is PVI a successful procedure for AF? The success of PVI in eliminating AF episodes can be attributed to a number of factors such as: 1) isolation of the trigger(s), 2) modification of the arrhythmogenic substrate located in the left atrial posterior wall, 3 ) interruption of crucial pathways of conduction, 4) atrial debulking or 5) atrial denervation.

It is generally assumed that the arrhythmogenic substrate for AF is located at the left atrial posterior wall. In humans, 
'rapid electrical activity' (organised electrograms with relatively short cycle lengths) during AF was found at the left atrial posterior wall. Recently, Shivkumar et al. tested the feasibility of Focal Impulse and Rotor Modulation (FIRM) by a novel method in 12 patients (paroxysmal AF: 1; persistent AF: 11) in a multicentre setting [12]. Unipolar AF electrograms were obtained by a 64-pole basket catheter (IE 4-8 $\mathrm{mm}$ ). In these patients, they identified 18 locations of 'rapid electric activity' in the left atrium and 5 in the right atrium; 21 were labelled as rotors. The procedural endpoint was defined as acute AF termination or organisation ( $>10 \%$ cycle length prolongation) and was conceived in all patients. FIRM demonstrated localised AF rotors/focal sources in patients with different AF types and recommended larger studies with follow-up and randomisation. Consequently, they initiated the Conventional ablation for atrial fibrillation with or without Focal Impulse and Rotor Modulation (CONFIRM) trial containing 92 patients (paroxysmal AF: 26; persistent AF: 66) undergoing 107 consecutive ablation procedures [13]. They allocated patients to FIRM following conventional ablation ( $N=36$; PAF: 7; persistent AF: 29) or conventional ablation alone $(N=71)$. In $97 \%$ of the procedures, they observed focal activity (left atrium $76 \%$; right atrium $24 \%$ ). Acute success was achieved in $86 \%$ of FIRM versus $20 \%$ of conventional ablation. In addition, patients with FIRM had a higher freedom of AF episodes $(82.4 \%$ vs. $44.9 \% ; p>0.001)$ during an averaged follow-up period of 273 days. Hence, it was claimed that FIRM-guided ablation might be a more efficient AF ablation approach for both paroxysmal and persistent AF.

Alterations in refractoriness may also contribute to PV arrhythmogenicity. Jais et al. assessed both the effective and functional refractory period in patients with and without a history of AF [14]. In patients with AF, the refractory period in the PV area was shorter than in the left atrium. On the contrary, in the control group the refractory period in the left atrium was shorter than in the PV area. Comparing the control and the AF group, the refractory period in the PV was significantly shorter in the AF patients. Hence, the relatively shorter refractory period of the $\mathrm{PV}$ area seems to contribute to its arrhythmogenicity. Haissaguerre et al. observed that AF cycle length within the coronary sinus progressively increased during PVI [15]. Based on their observations and data from other studies demonstrating reentry in the PV-left atrial junction region they stated that in some patients with AF, the PV-left atrial junction region is a generator of venous waves maintaining AF (the 'venous wave hypothesis') [16].

Disconnection of crucial intra-atrial conduction pathways, e.g. the Marshall bundle, located at the PV-left atrium junction, may also explain why PVI is successful in eliminating AF. The Marshall bundle is located within the vein of Marshall close to the left superior PV and connects the coronary sinus muscle sleeve to the left atrium. Epicardial mapping studies in canine atria demonstrated that the Marshall bundle may serve as a source of atrial ectopy giving rise to AF. Direct connections between the Marshall bundle and the left superior PV contributed to focal activity within the left superior PV. When conduction between the PV and left atrium was slowed by ablation, focal activation within the left superior PV was prevented, despite persistent focal activation originating from the Marshall bundle in some dogs. This finding also suggests that the Marshall bundle can be regarded as an accessory pathway to the left superior PV and may explain why circular lesions fail to isolate the PV. However, evidence from human data supporting this hypothesis is lacking [17].

Another hypothesis for the success of the PVI is atrial debulking. 'The critical mass of fibrillation' hypothesis states that a certain minimal size of atrial tissue is essential for initiation and sustenance of AF [18, 19]. Perpetuation of AF is determined by the number of simultaneously wandering wavelets [20]. Thus, a larger atrial mass can accommodate more wavelets, thereby stabilising AF and reduction of atrial mass may therefore be anti-fibrillatory. It is thought that PVI diminishes atrial mass as a significant amount of atrial myocardium may be replaced by scar tissue $[18,19]$.

Finally, effectiveness of the PVI can also be explained by atrial denervation. There is accumulating evidence that the autonomic nervous system plays a role in the pathogenesis of AF. Vagal stimulation increases spatial dispersion in atrial refractoriness, caused by e.g. local variability in density of vagal nerve endings/muscarine receptors, discrepancies in the coupling of muscarine receptors to ion-channel effectors, variation in $\mathrm{K}^{+}$-channel density or variations in the distribution of acetylcholinesterase. A higher vagal tone due reduced acetylcholinesterase activity has indeed been demonstrated in patients with AF [21]. Pappone et al. analysed heart rhythm variability and found a post-ablation shift of the sympathovagal balance towards parasympathetic predominance. In a subsequent study, vagal denervation was performed in 297 patients with paroxysmal AF referred for PVI. Vagal sites were defined as sites where vagal reflexes (bradycardia, asystole, atrioventricular block, hypotension) occurred during ablation. At such a site, ablation was performed until the vagal reflexes were completely abolished. Vagal reflexes were present in 104 patients; ablation sites were located at the cranial left superior PV-left atrium, the septal right superior PV-left atrium, the posteroinferior left inferior PV-left atrium and the posteroinferior right inferior PV-left atrium junction. In patients who have had a vagal denervation, late recurrences were less frequently observed though there was a higher incidence of early recurrences compared with patients without vagal denervation. This finding was explained by destruction of vagal nerve fibres resulting in release of acetylcholine causing early recurrences, followed by a stable vagolytic effect later after ablation which prevented AF recurrences [22]. 
Fig. 2 Principles of isochronal mapping. Upper panel: unipolar epicardial atrial electrogram recorded during sinus rhythm. Lower panel: unipolar epicardial atrial potentials recorded at every electrode $(N=60)$ within a mapping area of $1.10 \mathrm{~cm}^{2}$. Red lines indicate the local activation time at every electrode. A colourcoded activation map with isochrones (black lines) drawn at $10 \mathrm{~ms}$ shows that the wavefront is propagating from the lower right corner towards the upper left corner. $A$ atrial potential, $V$ far field ventricular potential


Fig. 3 Unipolar electrograms during induced and long-standing persistent atrial fibrillation. Unipolar, epicardial atrial electrograms recorded from the middle of the right atrial free wall during induced $\mathrm{AF}$ (upper panel) and long-standing persistent $\mathrm{AF}$ (lower panel). Recordings obtained from the patient with long-standing persistent $\mathrm{AF}$ are more complex, containing fractionated potentials

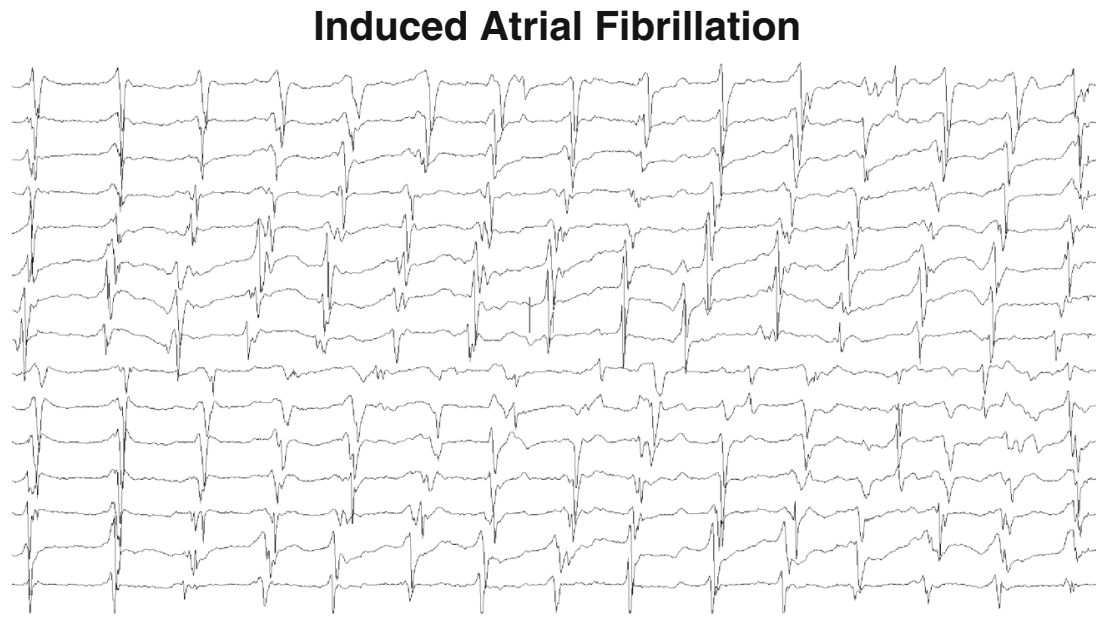

Long-Standing Persistent AtrialFibrillation



$200 \mathrm{~ms}$ 
So why and when may PVI then NOT be effective in eliminating AF? It is assumed that in patients with longstanding persistent atrial fibrillation, AF has progressed from a trigger-driven to a substrate-mediated arrhythmia. In these patients, persistence of AF no longer depends on the presence of a trigger but is maintained by an 'arrhythmogenic substrate' ('true fibrillation'). Patients presenting with AF may have underlying structural heart diseases affecting atrial architecture and/or electrical function thereby creating a substrate capable of perpetuating AF. In addition to this, AF itself may also modify the atrial myocardium which in turn stabilises AF. For example, AF results in shortening of the atrial effective refractory period and reversion of the physiological rate adaptation (shortening of the atrial refractory period at slower heart rates), facilitating inducibility and stability of AF (electrical remodelling) [23]. Longstanding AF in the goat model of AF is associated with changes in myocardial structure including dedifferentation, increase in cell size, perinuclear accumulation of glycogen, central loss of sarcomeres, changes in mitochondrial shape, fragmentation of the sarcoplasmatic reticulum and disorganisation of fibre orientation (structural remodelling) [24]. Unfortunately, data on histopathological alterations in humans with $\mathrm{AF}$ are limited [25].
There are numerous electrophysiological, structural and neurohormonal alterations creating a vulnerable substrate for AF. As it is likely that electrical and/or structural remodelling affects multiple sites of the atria, the arrhythmogenic substrate of AF will not only be confined to the PV area. This hypothesis is supported by the outcome of the IRAAF study (The Intraoperative Radiofrequency Ablation of Atrial Fibrillation) [26]. In this study, creation of linear lesions in the left atrium without PVI eliminated AF in more than $90 \%$ of the patients. Ablation of AF should either destroy the trigger mechanism or alter the substrate perpetuating AF. However, a selective ablation approach requires knowledge of the mechanisms underlying AF in the individual patient. Cardiac mapping may be a suitable tool to acquire knowledge of the arrhythmogenic substrate necessary for selection of the optimal ablation strategy.

\section{Cardiac mapping}

Cardiac mapping was originally defined as a method by which potentials recorded directly from the surface of the heart are spatially depicted as a function of time in an integrated manner

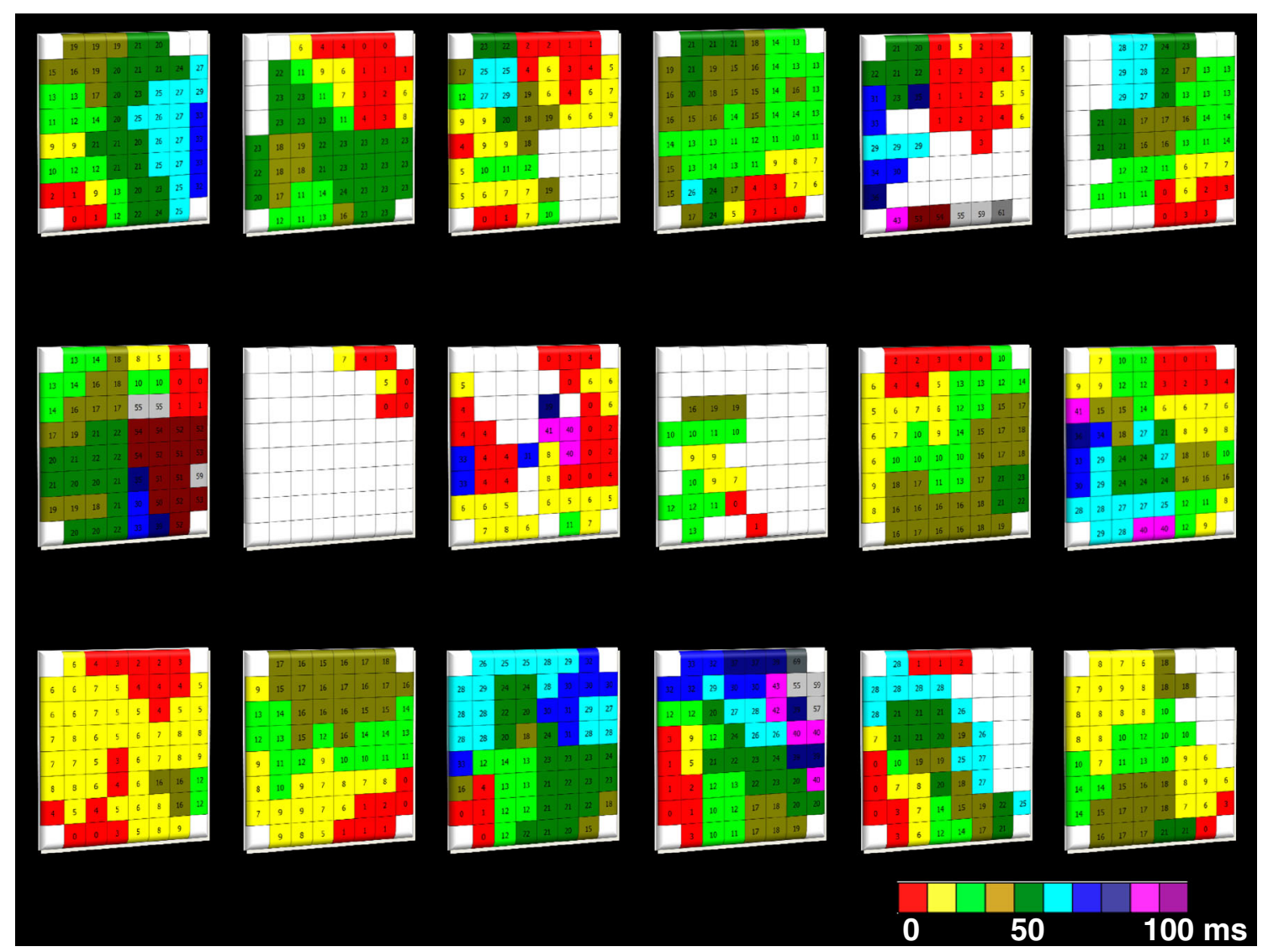

Fig. 4 Activation maps during permanent atrial fibrillation. Eighteen consecutive colour-coded activation maps of the right atrium (area $1.10 \mathrm{~cm} 2$ ) obtained from a patient with mitral valve disease. The maps show clear beat-to-beat changes in the pattern of activation. The earliest to latest activated areas are coloured from red to dark purple 
[27]. The most commonly used cardiac mapping approach is isochronal or activation mapping, which is aimed at creating a spatial model of the excitation sequence. Extracellular potential features are used to detect the local activation time of myocardium surrounding the recording electrode. Isochronal mapping during sinus rhythm is demonstrated in Fig. 2. A template containing 60 unipolar electrodes records extracellular potentials from a $1.10 \mathrm{~cm}^{2}$ area of epicardial atrial tissue at the right atrial free wall. An example of an unipolar atrial electrogram recorded during sinus rhythm, containing a local atrial potential (A) and a far-field ventricular potential (V), is shown at the top. Each potential in the electrogram has an identical morphology. The local activation time is determined

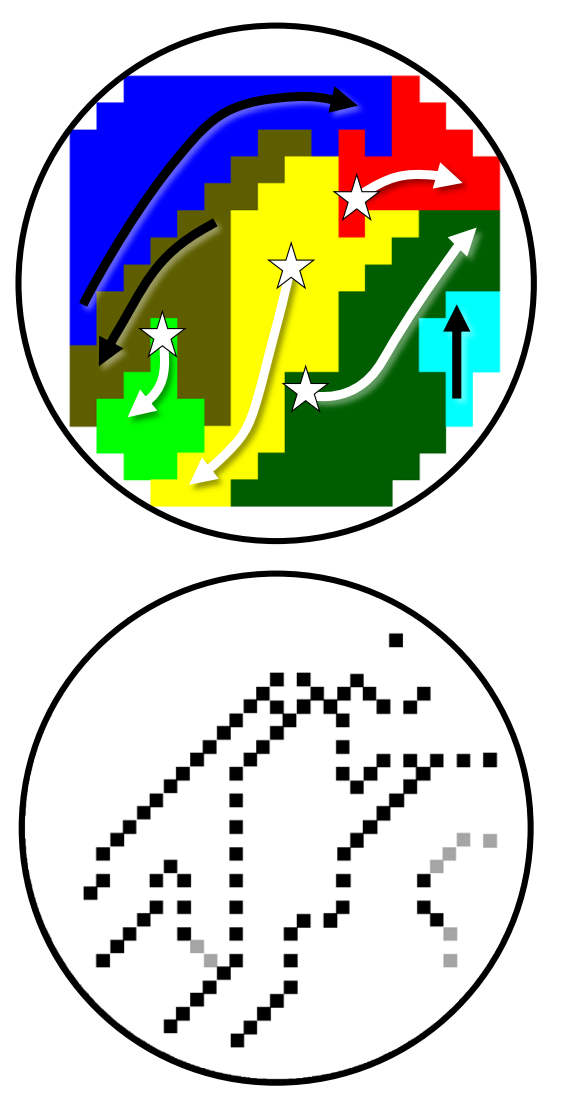

Fig. 5 Electropathological substrate of atrial fibrillation: longitudinal dissociation and epicardial breakthrough. Left panel: example of a wave map of the right atrial free wall of a patient with long-standing persistent $\mathrm{AF}$ and mitral valve disease. This wave map was reconstructed using an algorithm separating fibrillation waves with an interelectrode conduction time of $\geq 12 \mathrm{~ms}$ everywhere along their boundaries. The sequence of appearance of the waves is indicated by the rainbow colour scheme. Arrows indicate the main trajectories of the different fibrillation waves. These maps show two types of fibrillation wave: peripheral waves, entering the mapping area from outside the electrode array (black arrows) and epicardial breakthrough waves, appearing at the epicardial surface inside the mapping area (white arrows). Lower left panel: corresponding dissociation map demonstrating boundaries between waves with an interwave conduction velocity $\geq 19 \mathrm{~cm} / \mathrm{s}$ (grey boxes: collision) or $<19 \mathrm{~cm} / \mathrm{s}$ (black boxes, conduction block). Right panel: example of the spatial by marking the maximum negative slope (red lines). This information is used to construct a colour-coded activation map. The moment that the wavefront enters the mapping area is defined as $0 \mathrm{~ms}$. Isochrones are lines connecting simultaneously activated recording sites and are drawn at 10-ms intervals. The propagation direction of the wavefront is perpendicular to the isochrones and indicated by a black arrow. The resulting activation maps show a wavefront propagating from the lower right corner towards the upper left corner within $20 \mathrm{~ms}$.

As demonstrated by this example, the main element in cardiac mapping is thus correct interpretation of the morphology of extracellular potentials. Figure 3 shows a collection of



distribution of epicardial breakthroughs (EB) during $8 \mathrm{~s}$ of $\mathrm{AF}$ at the right atrial free wall. Each asterisk indicates a breakthrough site, its size being proportional to the number of EBs occurring at that site. EBs are widely distributed over the entire epicardial surface and occurred virtually everywhere. Lower right panel: schematic presentation of a pattern of $\mathrm{EB}$ as a result of transmural dissociation in conduction. For simplicity, transmural dissociation is represented by two planes only. In this example, the endocardial layer is activated by a single fibrillation wave propagating from left to right. In the middle, this endocardial wave is propagating transmurally through a junction site with the epicardial layer. Assuming a transmural conduction time of $10 \mathrm{~ms}$, the breakthrough of the transmurally propagating wave appeared at $t=25 \mathrm{~ms}$ at the epicardial surface (asterisk). Depending on the spatial distribution of electrophysiological properties such as strength of depolarisation, excitability, electric coupling, and source-sink relations, the spread of activation from this site of EB may vary [28, 29] 
unipolar, epicardial, AF electrograms recorded from the middle of the right atrial free wall during induced AF (upper panel) and long-standing persistent AF (lower panel). In both patients, there is a considerable beat-to-beat variability in the morphology of the fibrillation potentials that is more pronounced in the patient with longstanding persistent AF. Fibrillation potentials obtained from the latter patient are often prolonged and contain multiple deflections (so-called fractionated potentials). Mapping of AF is particularly challenging as these complex recordings hamper a correct determination of the local activation times. The hallmark of AF is a continuous beat-to-beat change in the pattern of activation (Fig. 4) and electrogram morphology. Thus, mapping of AF is very complex.

\section{QUASAR ( $Q$ Uest for the Arrhythmogenic $S$ ubstrate of $A$ trial fibRillation)}

In recent studies, Allessie et al. introduced a novel highresolution mapping approach ('wave mapping') to compare electrophysiological properties of fibrillation waves during induced atrial fibrillation in patients with normal atria (physiological AF) and during persistent AF in patients with valvular heart disease (pathological AF). This wave-mapping technique allows visualisation of individual fibrillation waves and quantification of the fibrillatory process. Epicardial wave maps of the right atrial free wall, left atrium and the PV region were constructed during persistent AF and compared with epicardial maps of the right atrial free wall obtained during induced AF. By applying this technique we were able to differentiate physiological from pathological AF and demonstrated that electrical dissociation of atrial muscle bundles (Fig. 5: left panel) and epicardial breakthrough of fibrillation waves (Fig. 5: right panel) play a key role in development of the substrate of persistent AF [28, 29].

Hypothetically, multisite high-resolution atrial mapping can be used to localise sources generating AF in patients with trigger-driven AF and to identify areas perpetuating AF in patients with substrate mediated-AF. Due to the large variety of disorders associated with AF, it is feasible to assume that there are inter-individual differences in the mechanisms underlying AF and multisite high-resolution mapping of the entire atria is therefore mandatory. In an attempt to diagnose the substrate of AF in individual patients, we initiated the QUASAR study (QUest for the Arrhythmogenic $\underline{\text { Substrate }}$


Rotterdam. In this project, we are developing a real-time, high-resolution, multisite epicardial mapping technique of the entire atria as a novel diagnostic tool. For this purpose, we are performing epicardial mapping studies in a large number of patients undergoing cardiac surgery with a variety of underlying structural heart diseases and different types of AF. This new mapping technique (Fig. 6) can be used not only to gain insights into the arrhythmogenic substrate of AF, but also to develop novel therapies or to improve existing treatment modalities.

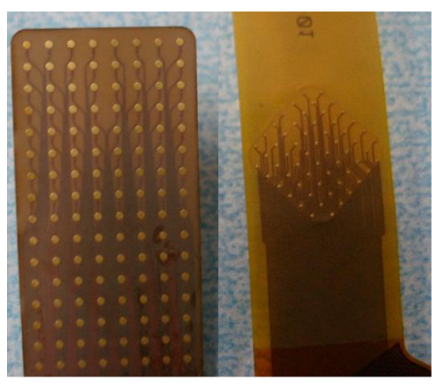

Signals

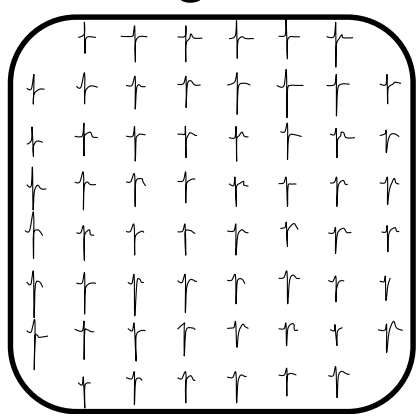

Voltages

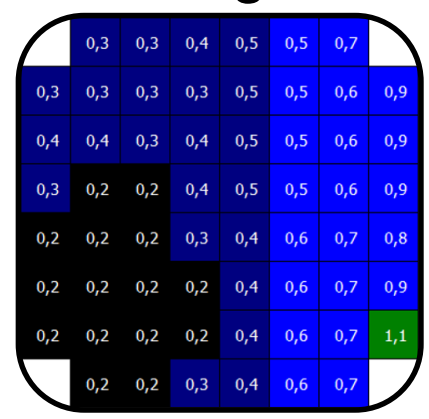

Fig. 6 Quest for the arrhythmogenic substrate of atrial fibrillation. An epicardial, high resolution, multi-site mapping approach is used for identification of the arrhythmogenic substrate in patients with AF. High



Activation

Conduction
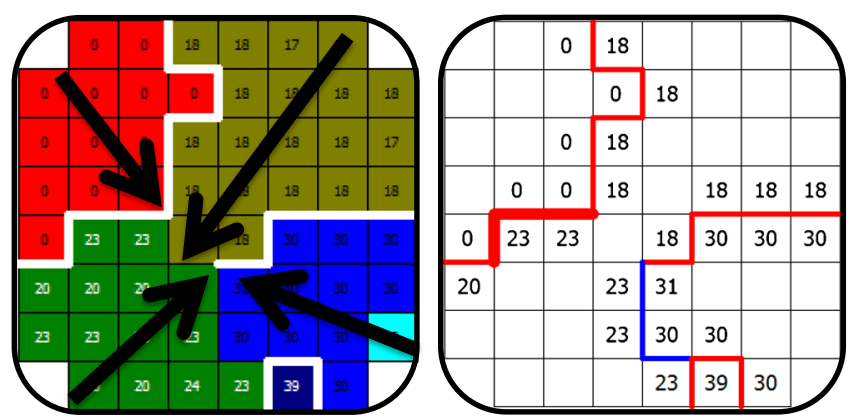

resolution mapping arrays (inter-electrode distances 1-2 mm) are used for epicardial mapping of the atria. Unipolar atrial potentials are used for construction of activation, voltage and conduction block maps 
Acknowledgments The authors would like to thank Drs. R. Bhagwandien for his contribution to Fig. 1.

Dr. NMS de Groot is supported by a grant from the EMC fellowship, Netherlands Heart Foundation and CoolSingel Foundation.

\section{Conflict of interests None declared.}

Open Access This article is distributed under the terms of the Creative Commons Attribution License which permits any use, distribution, and reproduction in any medium, provided the original author(s) and the source are credited.

\section{References}

1. Kannel WB, Abbott RD, Savage DD, et al. Epidemiologic features of chronic atrial fibrillation: the Framingham study. N Engl J Med. 1982;306:1018-22.

2. Levy S. Epidemiology and classification of atrial fibrillation. J Cardiovasc Electrophysiol. 1998;9:S78-82.

3. Peters NS, Schilling RJ, Kanagaratnam P, et al. Atrial fibrillation: strategies to control, combat, and cure. Lancet. 2002;359:593-603.

4. Psaty BM, Manolio TA, Kuller LH, et al. Incidence of and risk factors for atrial fibrillation in older adults. Circulation. 1997;96:2455-61.

5. Haissaguerre M, Shah DC, Jais P, et al. Mapping-guided ablation of pulmonary veins to cure atrial fibrillation. Am J Cardiol. 2000;86:9K$19 \mathrm{~K}$.

6. Camm AJ, Lip GYH, de Caterina R, et al. An update of the 2010 ESC Guidelines for the management of atrial fibrillation. Eur Heart J. 2012;33:2719-47.

7. Miyazaki S, Kuwahara T, Kobori A, et al. Long-term clinical outcome of extensive pulmonary vein isolation-based catheter ablation therapy in patients with paroxysmal and persistent atrial fibrillation. Heart. 2011;97:668-73.

8. Pappone C, Oreto G, Rosanio S, et al. Atrial electroanatomic remodeling after circumferential radiofrequency pulmonary vein ablation: efficacy of an anatomic approach in a large cohort of patients with atrial fibrillation. Circulation. 2001;104:2539-44.

9. Forleo GB, De Martino G, Mantica M, et al. Catheter ablation of atrial fibrillation guided by a 3D electroanatomical mapping system: a 2-year follow-up study from the Italian Registry On NavX Atrial Fibrillation ablation procedures (IRON-AF). J Interv Card Electrophysiol. 2013;37:87-95.

10. Kottkamp H, Tanner H, Kobza R, et al. Time courses and quantitative analysis of atrial fibrillation episode number and duration after circular plus linear left atrial lesions: trigger elimination or substrate modification: early or delayed cure? J Am Coll Cardiol. 2004;44:869-77.

11. Jons C, Hansen PS, Johannessen A, et al. The Medical ANtiarrhythmic Treatment or Radiofrequency Ablation in Paroxysmal Atrial Fibrillation (MANTRA-PAF) trial: clinical rationale, study design, and implementation. Europace. 2009;11:917-23.

12. Shivkumar K, Ellenbogen KA, Hummel JD, et al. Acute termination of human atrial fibrillation by identification and catheter ablation of localized rotors and sources: first multicenter experience of focal impulse and rotor modulation (FIRM) ablation. J Cardiovasc Electrophysiol. 2012;23:1277-85.
13. Narayan SM, Krummen DE, Shivkumar K, et al. Treatment of atrial fibrillation by the ablation of localized sources: CONFIRM (Conventional Ablation for Atrial Fibrillation With or Without Focal Impulse and Rotor Modulation) trial. J Am Coll Cardiol. 2012;60:628-36.

14. Jaïs P, Hocini M, Macle, et al. Distinctive electrophysiological properties of pulmonary veins in patients with atrial fibrillation. Circulation. 2002;106:2479-85.

15. Haïssaguerre M, Sanders $P$, Hocini M, et al. Changes in atrial fibrillation cycle length and inducibility during catheter ablation and their relation to outcome. Circulation. 2004;109:3007-13.

16. Haïssaguerre M, Sanders $P$, Hocini M, et al. Pulmonary veins in the substrate for atrial fibrillation: the 'venous wave' hypothesis. J Am Coll Cardiol. 2004;43:2290-2.

17. Okuyama Y, Miyauchi Y, Park AM, et al. High resolution mapping of the pulmonary vein and the vein of Marshall during induced atrial fibrillation and atrial tachycardia in a canine model of pacing-induced congestive heart failure. J Am Coll Cardiol. 2003;42:348-60.

18. Byrd GD, Prasad SM, Ripplinger CM, et al. Importance of geometry and refractory period in sustaining atrial brillation: testing the critical mass hypothesis. Circulation. 2005;112:I7-I13.

19. Jacquemet V, Virag N, Kappenberger L. Wavelength and vulnerability to atrial fibrillation: insights from a computer model of human atria. Europace. 2005;7:83-92.

20. Moe GK, Rheinboldt WC, Abildskov JA. A computer model of atrial fibrillation. Am Heart J. 1964;67:200-20.

21. Gonzalez RA, Campos EO, Karmelic C, et al. Acetylcholinesterase changes in hearts with sinus rhythm and atrial fibrillation. Gen Pharmacol. 1993;24:111-4.

22. Pappone C, Santinelli V, Manguso F. Pulmonary vein denervation enhances long-term benefit after circumferential ablation for paroxysmal atrial fibrillation. Circulation. 2004;109:327-34.

23. Wijffels MC, Kirchhof CJ, Dorland R, et al. Electrical remodeling due to atrial fibrillation in chronically instrumented conscious goats: roles of neurohumoral changes, ischemia, atrial stretch, and high rate of electrical activation. Circ. 1997;96:3710-20.

24. Ausma J, Wijffels M, Thoné F, et al. Structural changes of atrial myocardium due to sustained atrial fibrillation in the goat. Circulation. 1997;96:3157-63.

25. Wu TJ, Yashima M, Xie F, et al. Role of pectinate muscle bundles in the generation and maintenance of intra-atrial reentry: potential implications for the mechanism of conversion between atrial fibrillation and atrial flutter. Circ Res. 1998;83:448-62.

26. Kottkamp H, Hindricks G, Autschbach R, et al. Specific linear left atrial lesions in atrial fibrillation: intraoperative radiofrequency ablation using minimally invasive surgical techniques. J Am Coll Cardiol. 2002;40:475-80.

27. Berbari EJ, Landr P, Geselowitz DB. The methodology of cardiac mapping. In: Shenasa M, Borggrefe M, Breithard G, editors. Cardiac mapping. New York: Blackwell Publishing; 1993. p. 11-134.

28. Allessie MA, De Groot NMS, Houben RPM, et al. Electropathological substrate of long-standing persistent atrial fibrillation in patients with structural heart disease: longitudinal dissociation. Circ: AE. 2010;3: 606-15.

29. De Groot NMS, Houben RPM, Smeets JL, et al. Electropathological substrate of longstanding persistent atrial fibrillation in patients with structural heart disease: epicardial breakthrough. Circulation. 2010;122:1674-82. 